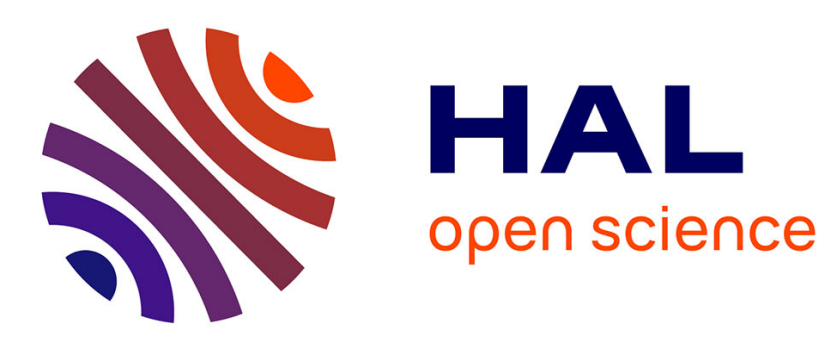

\title{
Gain Through Loss Frequency Comb Generation in Fiber Oscillators
}

A M Perego, F Bessin, M Conforti, A Kudlinski, S K Turitsyn, A Mussot

\section{To cite this version:}

A M Perego, F Bessin, M Conforti, A Kudlinski, S K Turitsyn, et al.. Gain Through Loss Frequency Comb Generation in Fiber Oscillators. ECOC 2020, Dec 2020, Brussels (virtual), Belgium. 10.1109/ECOC48923.2020.9333385 . hal-03442188

\section{HAL Id: hal-03442188 \\ https://hal.science/hal-03442188}

Submitted on 23 Nov 2021

HAL is a multi-disciplinary open access archive for the deposit and dissemination of scientific research documents, whether they are published or not. The documents may come from teaching and research institutions in France or abroad, or from public or private research centers.
L'archive ouverte pluridisciplinaire $\mathbf{H A L}$, est destinée au dépôt et à la diffusion de documents scientifiques de niveau recherche, publiés ou non, émanant des établissements d'enseignement et de recherche français ou étrangers, des laboratoires publics ou privés. 


\title{
Gain Through Loss Frequency Comb Generation in Fiber Oscillators
}

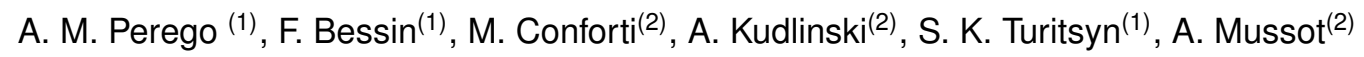 \\ (1) Aston Institute of Photonic Technologies, Aston University, Birmingham, B4 7ET, UK, \\ a.perego1@aston.ac.uk \\ (2) Univ. Lille, CNRS, UMR 8523-PhLAM Physique des Lasers Atomes et Molcules, F-59000 Lille, France
}

\begin{abstract}
We present a novel method to generate optical frequency combs in normal dispersion driven nonlinear resonators exploiting a filter induced modulation instability which enables selective waves amplification through losses. We demonstrate frequency comb generation in a fiber resonator with line spacing tuneability exceeding $100 \mathrm{GHz}$.
\end{abstract}

\section{Introduction}

Optical frequency combs consisting of a discrete set of equally spaced coherent frequency lines ${ }^{[1]}$ find a variety of applications from spectroscopy to distance ranging, from metrology to optical communications to name just a few. Being first demonstrated in mode-locked lasers, frequency comb generation has been achieved in nonlinear optical resonators too. In particular, optical microresonators constitute an ideal platform for portable optical frequency combs sources potentially integrable on a chip [2]. In Kerr nonlinear resonators, usually optical frequency combs are generated in the anomalous dispersion regime exploiting parametric amplification $[3$, or cavity solitons formation ${ }^{4}$, while in normal dispersion resonators optical frequency comb generation via dark solitons has been shown too [5]. In driven resonators the comb repetition rate is in general determined by the optogeometric properties of the cavity, namely by its free spectral range. Controlling the comb repetition may offer improved measurement sensitivity and at the same time allow to use the same source for different applications requiring different comb line spacing. Tuneability of the comb line spacing in driven optical resonators has been demonstrated for instance in the anomalous dispersion regime by scanning the pump wavelength $[$, exploiting the physics of soliton crystals ${ }^{77}$, using electronically controlled intracavity graphene devices $[\overline{6}$, and in media exhibiting quadratic nonlinearity by means of electro-optic modulation too 9 .

In this paper we present recent results about a novel method for generating optical frequency combs in normal dispersion passive driven nonlinear resonators which exploits dissipation induced modulation instability (MI) as a generation mech- anism[10],11], and requires simply the use of a tuneable pump laser and of an intracavity spectral filter.

\section{Theory}

The electric field dynamics inside a ring fiber resonator with group velocity dispersion $\beta_{2}$ and nonlinearity coefficient $\gamma$ is theoretically described using an Ikeda map approach: the propagation part is modelled by the nonlinear Schroedinger equation, and boundary conditions are applied after each cavity roundtrip:

$$
\begin{aligned}
& \frac{\partial A_{n}}{\partial z}=-i \frac{\beta_{2}}{2} \frac{\partial^{2} A_{n}}{\partial t^{2}}+\frac{\beta_{3}}{6} \frac{\partial^{3} A_{n}}{\partial t^{3}}+i \gamma\left|A_{n}\right|^{2} A_{n} \\
& A_{n+1}(z=0)=\theta \sqrt{P_{I N}}+\rho e^{i \phi_{0}} h(t) \star A_{n}
\end{aligned}
$$

where $A_{n}(z, t)$ is the electric field slowly varying envelope at roundtrip $n$, defined in a temporal reference frame co-moving with the pulse, and $0<z<L$ describes the spatial coordinate along the resonator. $\theta$ is the coupler transmission coefficient for the pump, $\rho$ is the coupler reflectivity, $\phi_{0}$ is the linear detuning, $P_{I N}$ the input pump power, $\beta_{3}$ is the third order dispersion coefficient, and $h(t)$ the filter response function, $\star$ denotes convolution. It is assumed that all the cavity losses are accounted for in the lumped coupler coefficient $\rho$ and that filter and coupler are located at the same position in the cavity. Due to physical causality, mathematically represented by the Kramers-Kronig relations, the filter function has a dispersive contribution too. For a complex filter response function defined in frequency domain as $\Psi_{f}=\exp \left(\alpha_{f}(\omega)+i \psi_{f}(\omega)\right)$, we calculated the dispersive contribution $\psi_{f}(\omega)$ from the attenuation profile by assuming a minimum phase filter for which $\psi_{f}(\omega)=\mathcal{F}\left\{\alpha_{f}(\omega)\right\}$ holds, where $\mathcal{F}$ is 
the Hilbert transform. The filter response function in time domain $h(t)$, can be calculated from $\Psi_{f}$ through Fourier transform. From Eqs. (1) it is possible to derive a phase-matching condition for the parametric process occurring in the nonlinear resonator, hence predicting the frequency of the amplified waves, and the MI gain too 111. Waves for which the following equation is satisfied will be amplified through the filter induced MI process:

$$
\frac{\beta_{2} \omega^{2}}{2} L+2 \gamma P L+\phi_{0}+\psi_{E}(\omega)=0
$$

where $\omega$ is the frequency detuning of the sidebands with respect to the pump, $L$ is the cavity length and $P$ is the intracavity power. As the mismatch parameter of the cavity depends significantly on the even part of the filter phase function $\psi_{E}(\omega)$, this physically means that the filter can be used to compensate the overall cavity mismatch arising due to normal dispersion and Kerr nonlinear phase shift. Parametric process can occur hence in normal dispersion regime and for zero cavity detuning (Turing stable regime). Filter induced $\mathrm{Ml}$ is hence a process which enables to obtain selective amplification -gain- for certain frequency from optical losses.

\section{Experimental results}

We have observed the filter induced $\mathrm{Ml}$ and associated OFC generation in an externally driven ring fibre resonator made of a $104.2 \mathrm{~m}$ long dispersion shifted fiber exhibiting normal dispersion $\left(\beta_{2}=0.5 \mathrm{ps}^{2} \mathrm{~km}^{-1}, \gamma=2.5 \mathrm{~W}^{-1} \mathrm{~km}^{-1}\right)$ at the pump wavelength $1544.66 \mathrm{~nm}$. The resonator has been pumped by square, $1.5 \mathrm{~ns}$ long pulses to avoid Brillouin scattering, and it was stabilized by an electronic feedback loop.

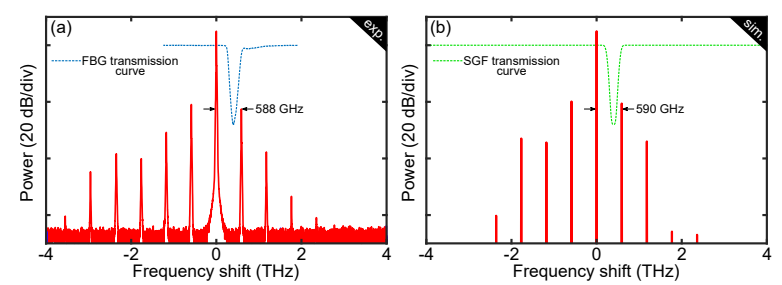

Fig. 1: OFC generated in experiment a) and in numerical simulations b). Dotted blue and green lines in a) and b) denote filter transmission profiles.

An intracavity fiber Bragg grating acting in reflection, detuned about $400 \mathrm{GHz}$ from the pump, having supergaussian shape, full width at half maxmimum around $330 \mathrm{GHz}$, and causing $28 \mathrm{~dB}$ attenuation at the maximum losses point, provided the required spectral filtering mechanism. The cavity detuning was chosen so that it com- pensated exactly the filter induced phase-shift at the pump wavelength, in this way Turing MI was avoided. After the primary sidebands were excited thanks to the filter induced Ml of the pump, a cascaded process led to the comb generation. The small number of comb lines that we observed was due to the low cavity finesse $(F=12)$; improving the cavity quality factor will result in the possibility of generating a very broad comb. By varying the detuning between the filter and the pump wavelength, which was possible by scanning the laser emission wavelength, we were able to show tuneability of the comb line spacing by more than $100 \mathrm{GHz}$.

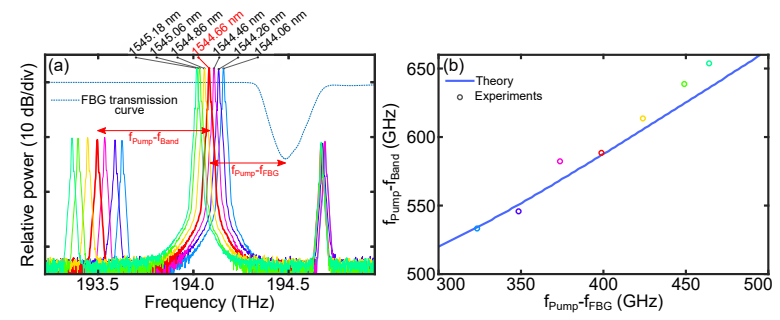

Fig. 2: Zoom on the pump and primary comb sidebands to show tuneability a), and comb line spacing versus pump-filter detuning b), dots are experimental points and continuous line theoretical predictions.

\section{Conclusions}

To conclude, we have shown how to exploit a filter induced $\mathrm{Ml}$ as the generation mechanism for optical frequency combs in normal dispersion nonlinear resonators with external driving. The peculiarity of this scheme consists in the possibility of tuning the comb repetition rate by simply varying the detuning between pump and filter position. We have shown proof-of-concept experimental results obtained in a ring fiber resonator. The approach presented in this paper could be potentially implemented to generate tuneable optical frequency combs at different wavelengths and in microresonators too.

\section{Acknowledgements}

The work of A.M.P. was supported by the Royal Academy of Engineering under the Research Fellowship scheme.

\section{References}

[1] T. W. Hänsch, "Nobel lecture: Passion for precision", Rev. Mod. Phys., vol. 78, pp. 1297-1309, 42006.

[2] J. T. Kippenberg, R. Holzwarth, and S. A. Diddams, "Microresonator-based optical frequency combs", Science, vol. 332, p. 555, 2011.

[3] P. Del'Haye et al., "Optical frequency comb generation from a monolithic microresonator", Nature, vol. 450, p. $1214,2007$. 
[4] T. J. Kippenberg, A. L. Gaeta, M. Lipson, and M. L. Gorodetsky, "Dissipative kerr solitons in optical microresonators", Science, vol. 361, no. 6402, 2018.

[5] X. Xue et al., "Mode-locked dark pulse kerr combs in normal-dispersion microresonators", Nat. Phot., vol. 9, p. 594, 2015.

[6] P. Del'Haye et al., "Octave spanning tunable frequency comb from a microresonator", Phys. Rev. Lett., vol. 107, p. $063901,2011$.

[7] D. Cole et al., "Soliton crystals in kerr resonators", Nat. Photon., vol. 11, p. 671, 2017.

[8] B. Yao et al., "Gate-tunable frequency combs in graphene-nitride microresonators", Nature, vol. 558, p. $410,2018$.

[9] M. Zhang et al., "Broadband electro-optic frequency comb generation in a lithium niobate microring resonator", Nature, vol. 568, p. 373, 2019.

[10] A. M. Perego, S. K. Turitsyn, and K. Staliunas, "Gain through losses in nonlinear optics", Light: Sci. Appl., vol. 7, p. 43, 2018.

[11] F. Bessin et al., "Gain-through-filtering enables tuneable frequency comb generation in passive optical resonators", Nat. Commun., vol. 10, p. 4489, 2019. 